\title{
Spectral properties of smart LED lamps
}

\author{
Jacek Kusznier*, Wojciech Wojtkowski \\ Faculty of Electrical Engineering, Bialystok University of Technology, Wiejska 45D, 15-351 Bialystok
}

Received February 27, 2020; accepted March 23, 2020; published March 31, 2020

\begin{abstract}
Intelligent LED lamps are most often used in intelligent lighting installations. They frequently use one of two different ways to obtain white light. The first is the mixing of monochrome lights $\mathrm{R}, \mathrm{G}$ and $\mathrm{B}$. The second way is to mix the light of the blue diode with the yellow phosphor radiation. Adjusting the luminous flux in RGBW LED lamps causes greater changes in the position of the chromaticity point than in the case of CW WW LED lamps. In the case of big changes, this can lead to visible change in the perceived color.
\end{abstract}

Depending on the time of year, time of day and the level of cloudiness, the sunlight reaching the Earth's surface is characterized by high spectral variability as well as variability in the illumination they cause. The human organism is adapted to such a rhythm of cyclical changes

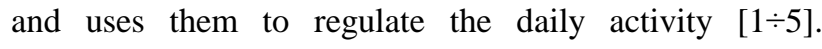
Lighting installations with smart light sources can adapt the lighting level and the emitted spectrum to this cycle. The group of smart light sources includes sources that allow the regulation of emitted luminous flux as well as correlated color temperature.

The exposure of an individual in the afternoon and evening hours to white light with a cold color, which helps us to maintain high activity during the day and reduces the amount of secreted melatonin, may result in problems with sleep and rest. These days we are unable to avoid the exposure to artificial light. Assessment of its impact on the human body requires knowledge of its spectral nature.

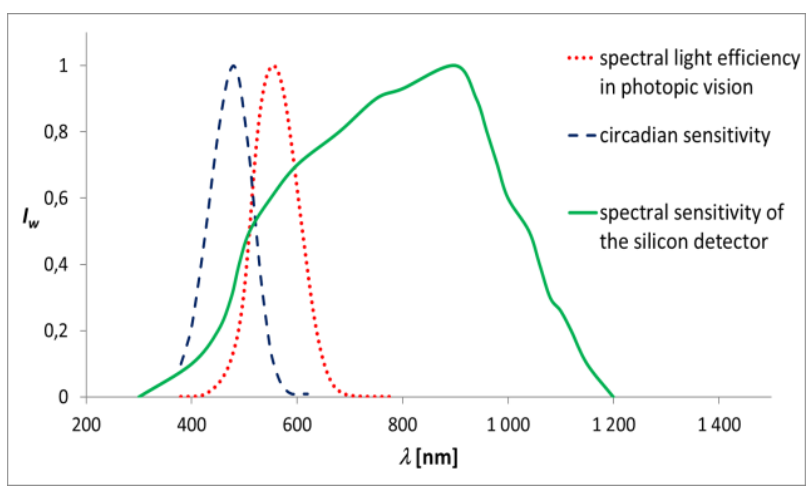

Fig. 1. Relative spectral characteristics: spectral light efficiency in photopic vision, circadian sensitivity and spectral sensitivity of the silicon detector (developed on the basis of $[6 \div 8]$ ).

${ }^{*}$ E-mail: j.kusznier@pb.edu.pl
Circadian effects are mainly caused by radiation in the $380 \mathrm{~nm}$ to $600 \mathrm{~nm}$ range. The strongest influence is observed for a wavelength of $460 \mathrm{~nm}$. It is a blue-colored radiation in the region of low spectral sensitivity in photopic vision. Therefore, we do not fully perceive this radiation with a force corresponding to its impact on the

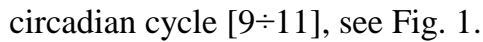

In order to adapt the level of lighting and the light spectrum to the natural daily circadian activity cycle, lighting installations with smart light sources are used. This group includes sources that give the ability to regulate the emitted light and change the correlated color temperature (spectral characteristics). At present, these are mainly sources based on LEDs [12].

In order to analyze the influence of light, the differences between the relative luminous efficacy curve for photopic vision, the circadian sensitivity curve and the relative sensitivity curve of the detector used should be considered $[9,13]$.

The correlated color temperature is determined, to the greatest extent, by the proportion of blue and red radiation $[14 \div 17]$. It should be emphasized, however, that sources with the same correlated color temperature and color rendering index often have significantly different spectral characteristics, see Fig. 2.

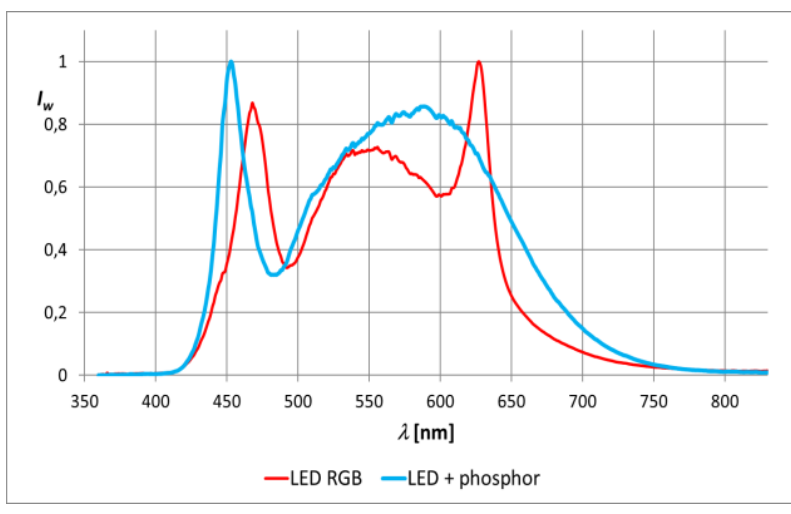

Fig. 2. Relative spectral characteristics of sample sources with $\mathrm{Tc}=5500 \mathrm{~K}$ (based on own research).

Light sources were tested, enabling the regulation of both the value of emitted luminous flux and correlated color temperature. The StellarNet Blue-Wave UVIS-50 spectrometer was used for the measurements. 
The first of the sources studied is the Osram Lightify Classic A 60 RGBW E27 lamp in which the light of a given spectral character is obtained by mixing RGB and supplemented by the light of a white diode. Increasing the set value of correlated color temperature resulted in a gradual increase in emission in the range of green and blue radiation (see Fig. 3). The result is a shift of the point on the CIE 1931 chromaticity plot (Fig. 4). Adjusting the value of the emitted luminous flux at the same preset color temperature caused changes in the spectral characteristics (Fig. 5), as well as changes in the location of the point and the MacAdam's ellipses on the chromaticity chart (Fig. 6).

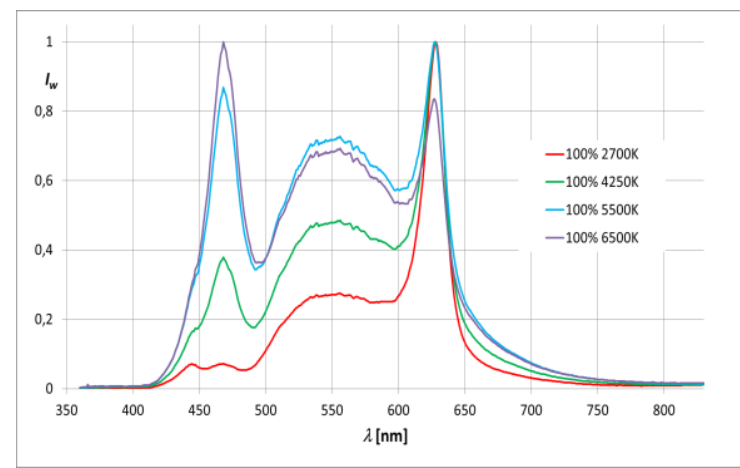

Fig. 3. Relative spectral characteristics of the Osram Lightify Classic A 60 RGBW E27 source at different Tc and luminous flux emitted in $100 \%$ (based on own research).

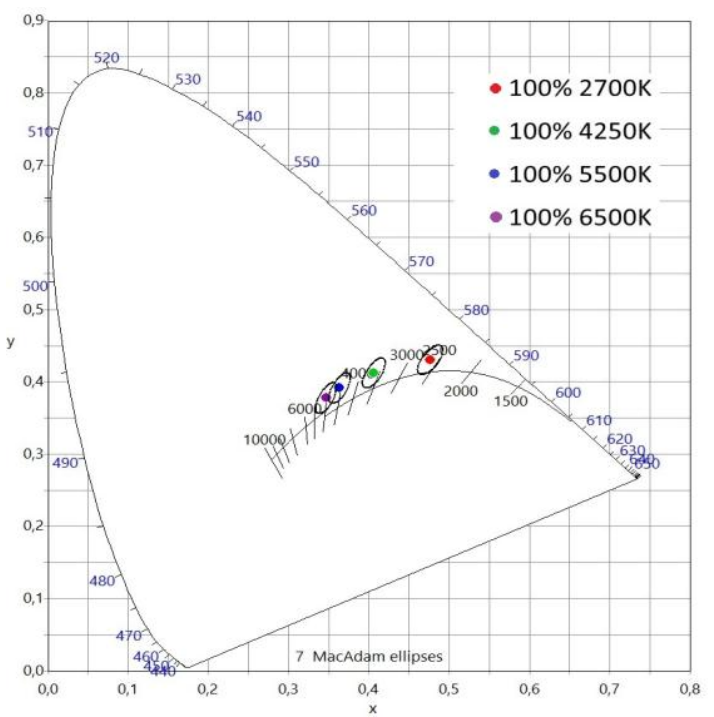

Fig. 4. Change in the position of the point on the chromaticity graph, Osram Lightify Classic A 60 RGBW E27 source at different Tc and luminous flux emitted in 100\% (based on own research).

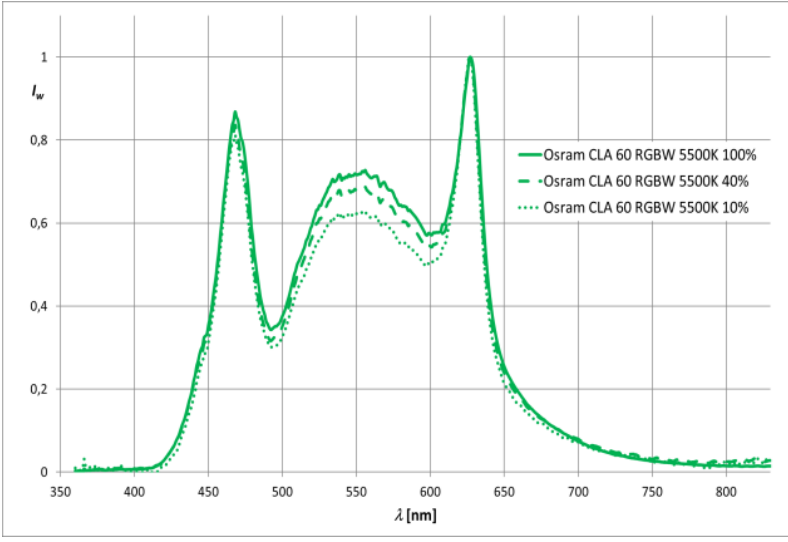

Fig. 5. Relative spectral characteristics of Osram Lightify Classic A 60 RGBW E27 source depending on the size of emitted luminous flux (based on own research).

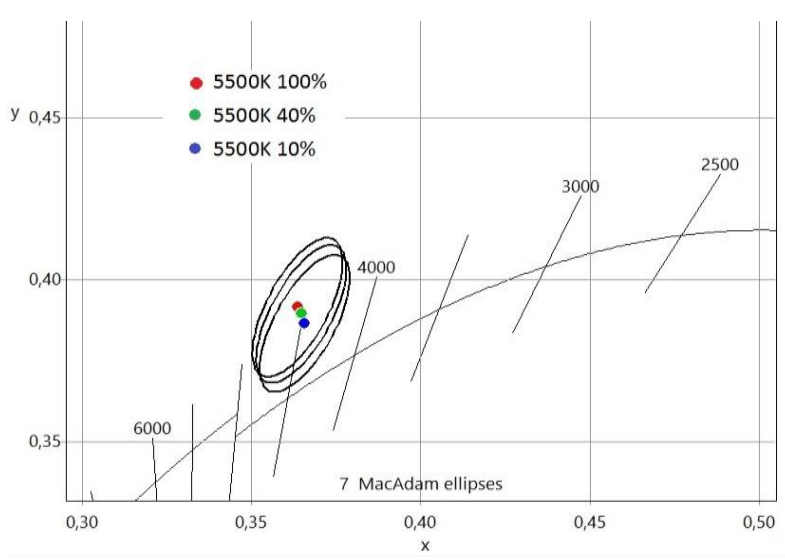

Fig. 6. Change in the position of a point on the chromaticity graph, Osram Lightify Classic A 60 RGBW E27 source depending on the size of emitted luminous flux (based on own research).

The second source studied was the Osram Lightify Classic A 60 TW E27 lamp. The change in the color temperature of this lamp is obtained by properly mixing the light streams of the CW (cold white) and WW (warm white) LEDs used. This allows for smooth regulation of the emitted light beam and color temperature. Changing the preset Tc value causes appropriate control of $\mathrm{CW}$ and WW diodes and thus a change in the spectral nature of the emitted light (Figs. $7 \div 8$ ). The adjustment of the level of the emitted light beam in this case did not cause any visible changes in the course of spectral characteristics, point location and MacAdam ellipses on the chromaticity graph (Figs. $9 \div 10$ ).

At present, only smart LED lamps allow to shape the spectral parameters in such a wide range. This allows to imitate natural sunlight changes. 


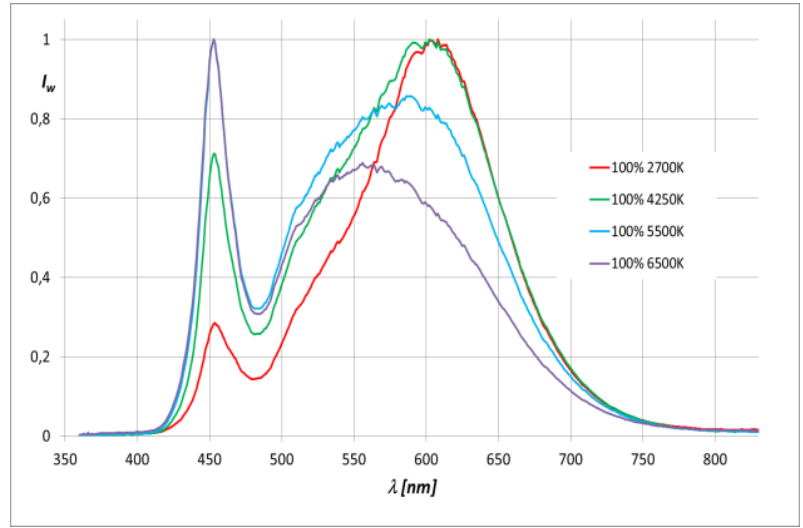

Fig. 7. Relative spectral characteristics of Osram Lightify Classic A 60 TW E27 source at different Tc and luminous flux emitted in 100\% (based on own research).

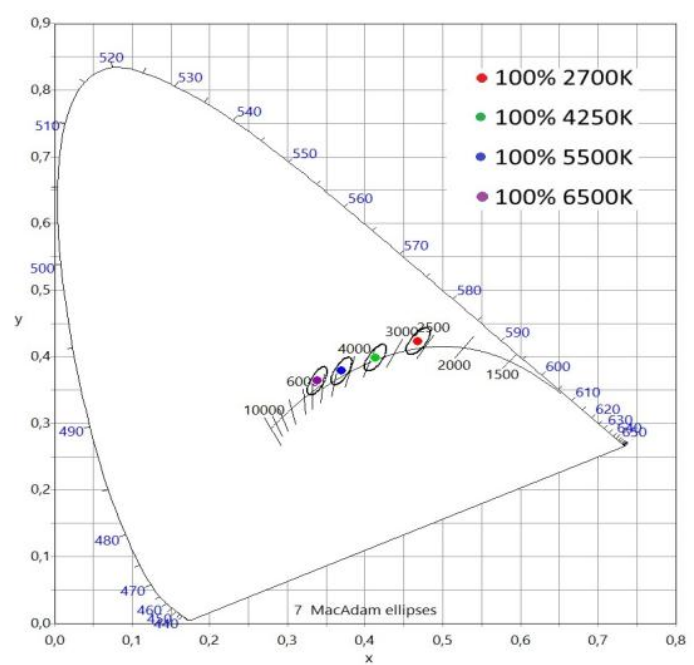

Fig. 8. Change in the position of the point on the chromaticity graph, Osram Lightify Classic A 60 TW E27 source at different Tc and luminous flux emitted in $100 \%$ (based on own research).

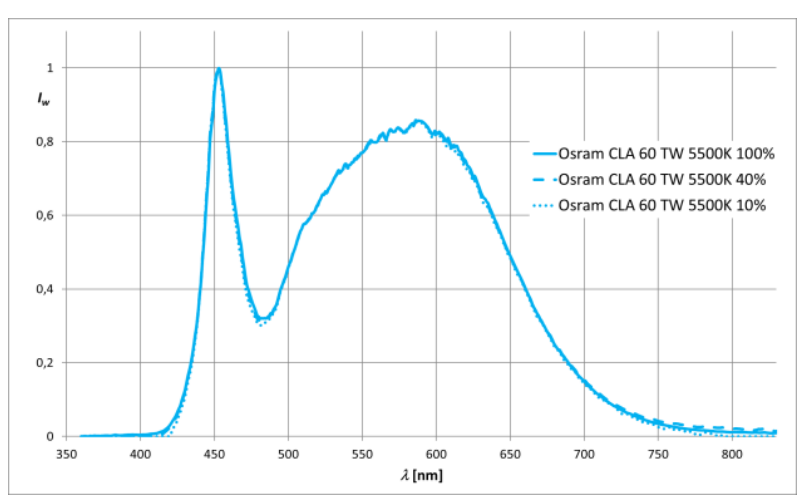

Fig. 9. Relative spectral characteristics of Osram Lightify Classic A 60 TW E27 source depending on the size of emitted luminous flux (based on own research).

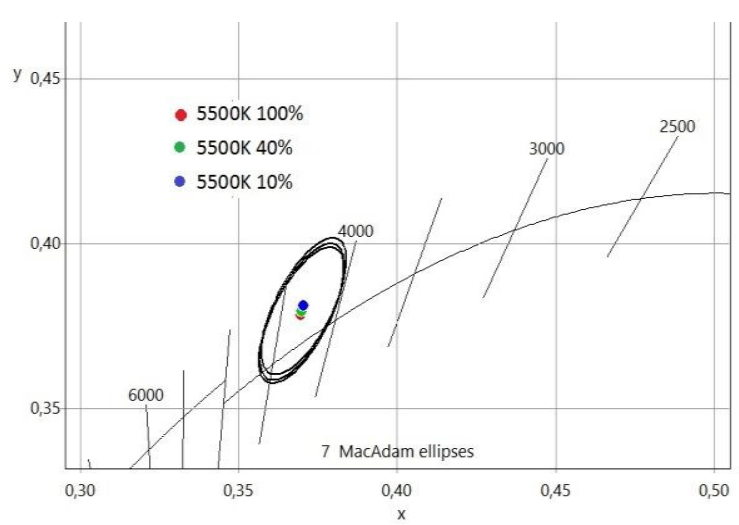

Fig. 10. Change in the position of the point on the chromaticity graph, Osram Lightify Classic A 60 TW E27 source depending on the size of emitted luminous flux (based on own research).

The research was conducted at the Bialystok University of Technology within the framework of Subject S/WE/3/2018.

\section{References}

[1] https://re.jrc.ec.europa.eu/pvg_tools/en/tools.html

[2] https://dateandtime.info/pl

[3] J. Kusznier, W. Wojtkowski, Impact of climatic conditions on PV panels operation in a photovoltaic power plant, IEEE, 2019 15th Selected Issues of Electrical Engineering and Electronics (WZEE), Zakopane, Poland (2019).

[4] J. Kusznier, W. Wojtkowski, Impact of climatic conditions and solar exposure on the aging of PV panels, IEEE, 2019 15th Selected Issues of Electrical Engineering and Electronics (WZEE), Zakopane, Poland (2019).

[5] https://www.weatheronline.pl/weather/

[6] L.T. Sharpe, A. Jagla, W. Jägle, J. Vision 5(11), 948 (2012).

[7] http://www.ee.co.za/article/integrating-sphere-data-sincere.html

[8] M.P. Simunovic, Arch. Ophthalmol. 130(7), 919 (2012).

[9] I. Fryc, Przeglad Elektrotechniczny R. 88(4a), 230 (2012).

[10] I. Fryc, Przeglad Elektrotechniczny R. 85(11), 317 (2009).

[11] J. Kowalska, I. Fryc, Przeglad Elektrotechniczny R. 95(7), 94 (2019).

[12] M. Gilewski, L. Gryko, A. Zajac, Proc. of SPIE 8902, 89021D (2013).

[13] M. Gilewski, Photon. Lett. Poland 11(3), 87 (2019).

[14] I. Fryc, T. Dimitrova-Grekow, An automated system for evaluation of the quality of light sources, 2016 IEEE Lighting Conference of the Visegrad Countries (Lumen V4), Karpacz, Poland (2016).

[15] I. Fryc, Przeglad Elektrotechniczny R. 88(6), 131 (2012).

[16] I. Fryc, Przeglad Elektrotechniczny R. 86(10), 187 (2010).

[17] J. Kusznier, Changes in the Spectral Power Distribution of Light Sources for Smart Lighting, IEEE 14th Selected Issues of Electrical Engineering and Electronics (WZEE), Szczecin, Poland (2018). 